\title{
Pilonidal sinus disease can be treated with crystallized phenol using a simple three-step technique
}

\author{
Mehmet Eren Yuksel ${ }^{1 凶}$
}

\begin{abstract}
Introduction: Pilonidal sinus disease can be treated with various surgical techniques. However, invasive surgical techniques harbor the risk of wound infection and require a long postoperative wound healing period. The treatment of pilonidal sinus disease with crystallized phenol is minimally invasive with a short postoperative recovery period. This article explains how to treat pilonidal sinus disease with crystallized phenol using a quick and easy three-step technique.

Methods: Between August 2014 and January 2016, 43 male and 7 female patients with pilonidal sinus disease were treated with crystallized phenol. Hair was gently removed from the sinus openings with a mosquito clamp. Afterwards, the pilonidal sinus openings were gently filled with crystallized phenol.

Results: The closure of pilonidal sinus openings without any leakage after a single application of crystallized phenol was accepted as healing. The treatment failed in two of the female patients, who had had prior pilonidal sinus surgery, and in four of the male patients, who had a recurrence after wound site infection. The overall cure rate was $88 \%$.

Conclusion: Treatment of pilonidal sinus disease with crystallized phenol using the three-step technique is simple, fast, and comfortable. Therefore, crystallized phenol should be preferentially considered in the treatment of pilonidal sinus disease.
\end{abstract}

Keywords: crystallized phenol, pilonidal sinus disease

Received: 5 December 2016 | Returned for modification: 6 January 2017 | Accepted: 30 January 2017

\section{Introduction}

Pilonidal sinus disease is a chronic disorder with sinus openings, hair in the gluteal cleft, subcutaneous tracts, and persistent drainage (1). It is a member of the follicular occlusion tetrad, which also includes hidradenitis suppurativa, acne conglobata, and dissecting cellulitis of the scalp (2). Follicular occlusion in apocrine gland-bearing areas is thought to initiate the pathological process causing the disease. Pilonidal sinus disease can be treated with crystallized phenol. This article explains how to treat pilonidal sinus disease with crystallized phenol using a quick and easy three-step technique in outpatient clinic settings.

\section{Methods}

\section{Preparation prior to application of crystallized phenol}

Excess hair is the main factor causing pilonidal sinus disease. Pilonidal sinus disease is a common condition in Middle Eastern countries. However, it is a rare condition in Nordic countries, where body hair is sparse. Therefore, hair removal is recommended prior to the application of crystallized phenol. Hair removal not only eliminates the source of the disease, but also opens a clean working area for the surgeon. Hair removal may also reduce the risk of wound infection after crystallized phenol treatment. Moreover, washing the anogenital area with hot water after hair removal is also recommended because it decreases the number of bacteria that might cause postoperative wound infection.

\section{Precautions to take prior to application of crystallized phenol}

Crystallized phenol (Phenol, Emprove ${ }^{\circledR}$, Merck, Darmstadt, Germany) is a white crystalline substance resembling granulated sugar. Crystallized phenol should be kept in glass bottles. Sun- light should be strictly avoided. When crystallized phenol comes into direct contact with the skin, it causes small white blisters. Therefore, a face mask, eyeglasses, gloves, and a surgical gown with long sleeves should be worn prior to phenol application. An intravenous route is not a must; however, a peripheral intravenous cannula should be inserted prior to the procedure to be used in case of an emergency.

\section{Step 1: Local anesthesia}

Patients should be questioned about their allergies and sensitivity to chemicals prior to application of lidocaine, nitrofurazone ointment, and crystallized phenol. Afterwards, the intergluteal region should be prepped and draped in a sterile manner. Local anesthetic ( $2 \%$ lidocaine solution) should be applied subcutaneously. Application of a local anesthetic is not mandatory; however, it increases patient comfort by decreasing pain sensation during the removal of hair from the pilonidal sinus pits.

\section{Step 2: Hair removal}

Hair should be gently removed from the sinus openings with the help of a mosquito clamp. Some patients might have bunches of hair in the sinus openings whereas others may not have any. The mosquito clamp should be used meticulously without applying excessive force. Applying excessive force in order to search for and collect all hair in the sinus openings might result in bleeding. If bleeding occurs, a gauze pad should be compressed until the bleeding stops spontaneously.

\section{Step 3: Application of crystallized phenol}

Crystallized phenol is solid at room temperature. However, crystallized phenol becomes liquid when it is applied to the skin. 
Because the liquid phenol may leak into the anus, the anus should be covered and protected with a gauze pad dipped in soluble dressing ointment. For this purpose, we use $0.2 \%$ nitrofurazone ointment (Furacin, Zentiva); however other ointments might also be applied. Crystallized phenol resembles sugar granules and these granules can be handled with a mosquito clamp. Crystallized phenol should be gently placed into the pilonidal sinus openings. The sinus openings should be filled with crystallized phenol. Crystallized phenol reacts with the skin and creates a blackish-dark brown color. As the crystallized phenol causes chemical debridement, blackish-dark brown leakage occurs from the sinus openings (Fig. 1). The leakage should be continuously cleared with a gauze pad. The gauze pad should always be kept in sight because any leakage towards the anus should be prevented immediately. After the treatment, a gauze pad is placed in the intergluteal sulcus. The gauze pad will absorb excessive leaking liquid phenol and debris. The amount of crystallized phenol to use for filling pilonidal sinus openings and the number of crystallized phenol application cycles has not been standardized yet. Therefore, the surgeon should try to finish the chemical debridement with the least amount of crystallized phenol needed.

\section{Results}

Between August 2014 and January 2016, 43 male and 7 female patients with pilonidal sinus disease were treated with crystallized phenol using the three-step technique described above at Devrek State Hospital, Department of General Surgery. The mean age of the patients was 27 (range: 13-50). The closure of pilonidal sinus openings without any leakage after a single application of crystallized phenol was accepted as healing. The mean postoperative healing time was 30 days (range: 14 to 45 days). The treatment failed in two of the female patients, who had had prior pilonidal sinus surgery, and in four of the male patients, who had a recurrence after wound site infection. The overall cure rate was $88 \%$.

\section{Discussion}

Pilonidal sinus disease can be treated with various surgical techniques such as excision and primary closure, rhomboid excision
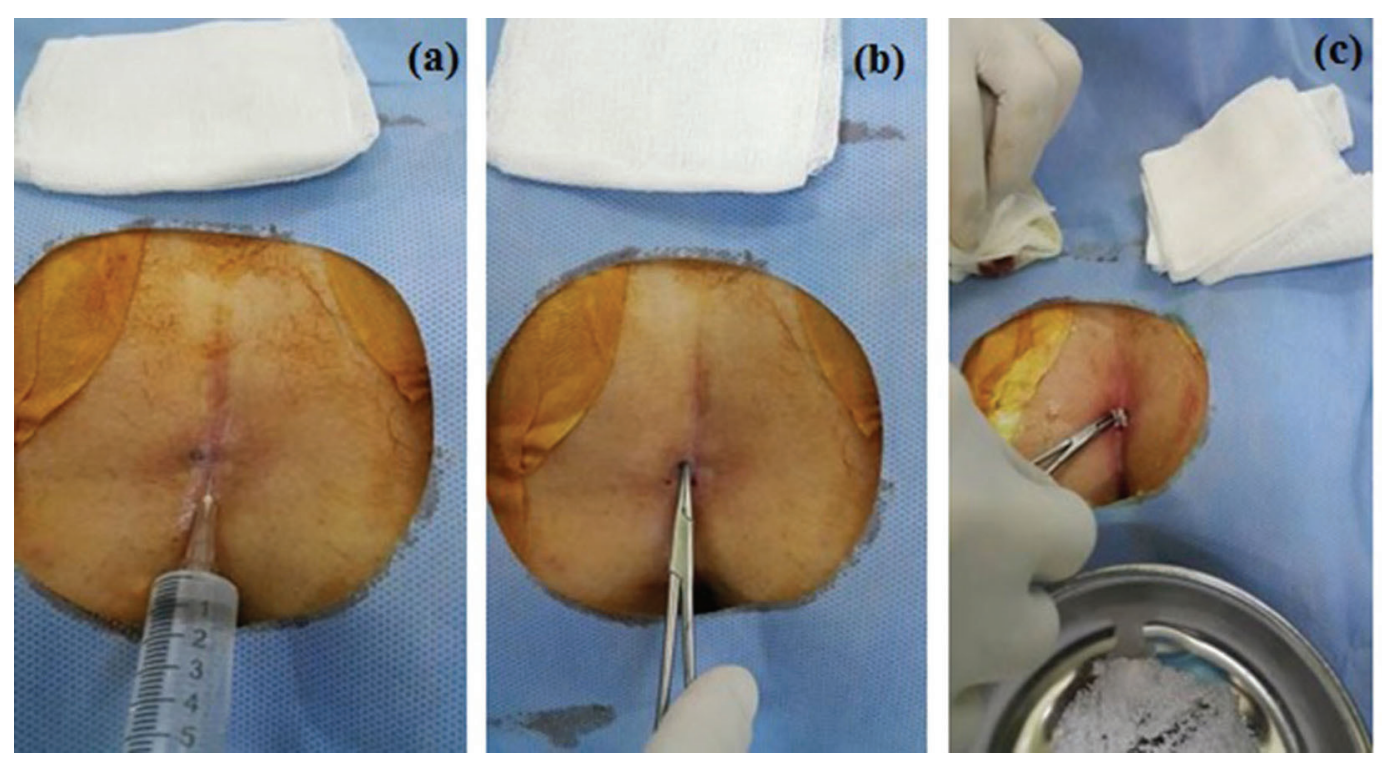

and Limberg flap, marsupialization, and cleft lift procedure. However, all of these invasive surgical techniques harbor the risk of wound infection and require a long postoperative wound healing period. Nevertheless, the treatment of pilonidal sinus disease with crystallized phenol is minimally invasive with a short postoperative recovery period.

There are numerous advantages of pilonidal sinus disease treatment with crystallized phenol. Crystallized phenol is inexpensive and its application is simple (2). Prior to application of crystallized phenol, no preoperative laboratory tests are mandatory. Only hemoglobin, prothrombin time, partial thromboplastin time, and INR values should be considered to exclude any prior coagulopathy. There is no need for preoperative anesthesia consultation, distal bowel cleansing, or prophylactic antibiotics prior to application of crystallized phenol.

The treatment of pilonidal sinus disease with crystallized phenol is minimally invasive. There is no need for surgery room settings. Therefore, the physician can apply the crystallized phenol alone without any need for further assistance in outpatient clinic settings. Treatment with crystallized phenol improves quality of life in patients with pilonidal sinus disease (3). After the application of crystallized phenol, the patients can sit normally on a chair and on the toilet without any precautions. Moreover, the patients are allowed to travel after the procedure. Compared to surgical treatment of pilonidal sinus with excision and primary closure, crystallized phenol treatment results in less perioperative pain and a shorter postoperative rest period. There is no need to remain in the hospital because patients may return to their normal daily activities after the procedure. Wound care is not complicated because no wound dressing is needed. In addition to the patients' swift recovery, the cosmetic results are excellent. Because no stitches or drains are used, there is no surgical scar after treatment. The closure of sinus openings without any leakage is accepted as healing.

Possible postoperative complications are mild pain, discomfort, purulent discharge, and abscess formation. However, these complications can be treated with non-steroidal anti-inflammatory drugs and antibiotics such as ciprofloxacin in combination with metronidazole.

Crystallized phenol application is an effective treatment option

Figure 1 | Pilonidal sinus disease can be treated with crystallized phenol using a simple three-step technique. a. Step 1: Local anesthetic (2\% lidocaine solution) is applied subcutaneously. Application of the local anesthetic is not mandatory; however, it increases patient comfort by decreasing pain sensation during the removal of hair from the pilonidal sinus opening. b. Step 2: Hair is removed gently with a surgical clamp from the pilonidal sinus pit. c. Step 3: Crystallized phenol is applied gently through the sinus opening. d. Postoperative view. The total procedure time is around 10 minutes. The patient can be discharged and return home after the treatment. 
with low recurrence rates. The 44 of 50 patients with pilonidal sinus disease treated with three-step crystallized phenol technique at Devrek State Hospital healed completely with an $88 \%$ cure rate. The 6-month follow-up of the patients revealed no recurrences. However, Girgin et al. achieved a cure rate of $64.5 \%$ in patients with pilonidal sinus disease after a single phenol application and a cure rate of $95 \%$ with two or more applications. Girgin et al. did not observe any recurrence during 22 months of follow-up (4). Furthermore, Kaymakcioglu et al. applied 80\% phenol to 143 patients with pilonidal sinus disease and recurrence was observed in only $12(8.2 \%)$ patients during 2 years of follow-up (5).

Moreover, Akan et al. compared the efficacy of the crystallized phenol method with the surgical Limberg flap technique in pilonidal sinus treatment (6). The average length of hospital stay in the Limberg group was $1.46 \pm 0.61$ days; however, all of the patients in the crystallized phenol group were discharged on the same day. Infection, hematoma, wound dehiscence, and cosmetic problems were significantly higher in the Limberg group. There was no difference between the two groups in terms of recurrence. Calıkoglu et al. compared phenol injection versus excision with

\section{References}

1. Steele SR, Perry WB, Mills S, Buie WD. Standards practice task force of the American Society of Colon and Rectal Surgeons. Practice parameters for the management of pilonidal disease. Dis Colon Rectum. 2013;56:1021-7.

2. Vasanth V, Chandrashekar BS. Follicular occlusion tetrad. Indian Dermatol Online J. 2014;5:491-3.

3. Topuz O, Sözen S, Tükenmez M, Topuz S, Vurdem ÜE. Crystallized phenol treatment of pilonidal disease improves quality of life. Indian J Surg. 2014;76:81-4.

4. Girgin M, Kanat BH. The results of a one-time crystallized phenol application for pilonidal sinus disease. Indian J Surg. 2014;76:17-20. the open healing technique (7). Time to complete wound healing $(16.2 \pm 8.7$ versus $40.1 \pm 9.7$ days) was significantly in favor of the phenol injection group $(\mathrm{p}<0.001)$. The median operation time was $14.0 \pm 3.8$ minutes in the phenol group versus $49.0 \pm 24.2 \mathrm{~min}-$ utes in the excision with open healing group $(\mathrm{p}<0.001)$. At the mean follow-up of $39.2 \pm 9.0$ months after surgery, there was no difference in the recurrence rate between the treatment arms.

In conclusion, treatment of pilonidal sinus disease with crystallized phenol using the three-step technique is simple, fast, and comfortable with high cure rates. Therefore, crystallized phenol should be preferentially considered in the treatment of pilonidal sinus disease.

\section{Acknowledgement}

I would like to express my sincere gratitude to Prof. Dr. Sezai Leventoglu, Gazi University School of Medicine, Department of General Surgery, for his guidance in treating pilonidal sinus disease with crystallized phenol. His commitment to improving the crystallized phenol technique made it possible to write this article.
5. Kaymakcioglu N, Yagci G, Simsek A, Unlu A, Tekin OF, Cetiner S, et al. Treatment of pilonidal sinus by phenol application and factors affecting the recurrence. Tech Coloproctol. 2005;9:21-4.

6. Akan K, Tihan D, Duman U, Özgün Y, Erol F, Polat M. Comparison of surgical Limberg flap technique and crystallized phenol application in the treatment of pilonidal sinus disease: a retrospective study. Ulus Cerrahi Derg. 2013;29:162-6.

7. Calikoglu I, Gulpinar K, Oztuna D, Elhan AH, Dogru O, Akyol C, et al. Phenol injection versus excision with open healing in pilonidal disease: a prospective randomized trial. Dis Colon Rectum. 2017;60:161-9. 